\title{
Association Between Elevated Cardiac Troponin Level and Clinical Outcomes in Patients with Infective Endocarditis: A Meta-Analysis
}

\section{Yunfei Ling}

Sichuan University West China Hospital

\section{Zheng Chai}

Sichuan University West China Hospital

\section{Tiange Li}

Sichuan University West China Hospital

\section{Zhongze Cao}

Sichuan University West China Hospital

\section{Kerun Chen}

Sichuan University West China Hospital

\section{Yongjun Qian ( $\nabla$ qianyongjun@scu.edu.cn )}

West China Hospital https://orcid.org/0000-0002-8013-4070

\section{Research article}

Keywords: infectious endocarditis, elevated cardiac troponin, meta-analysis, prognosis

Posted Date: February 9th, 2021

DOl: https://doi.org/10.21203/rs.3.rs-200322/v1

License: (1) This work is licensed under a Creative Commons Attribution 4.0 International License. Read Full License 


\section{Abstract}

Background: Infective endocarditis is a serious infection associated with high mortality. Timely intervention is paramount to achieve a favorable prognosis in patients with Infective endocarditis. At present, the correlation between cardiac troponin level and Infective endocarditis prognosis is not well established.

Methods: Pubmed, Embase, and Cochrane databases were systematically searched for studies examining the relationship between elevated serum cardiac troponin concentration and prognosis in patients with IE. Literature screening, data extraction and quality appraisal were undertaken by two independent reviewers.

Results: A total of 7 relevant studies were included in this study. Patients with elevated troponin were significantly associated with higher incidences of in-hospital mortality [OR=5.87, 95\% $\mathrm{Cl}(3.37,10.21)]$, one-year mortality [OR=3.28, 95\% $\mathrm{Cl}(1.01,10.62)]$, surgery or valve replacement $[\mathrm{OR}=2.18,95 \% \mathrm{Cl}$ $(1.36,3.51)]$, symptoms of central nervous system (CNS) [OR=3.28, 95\% $\mathrm{Cl}(1.01,10.62)]$, and cardiac abscesses [OR=3.28, 95\% $\mathrm{Cl}(1.01,10.62)]$. There was no significant correlation between elevated troponin and incidence of renal failure, embolization, or cerebrovascular events in patients with Infective endocarditis.

Conclusion: Elevated cardiac troponin in patients with Infective endocarditis is associated with higher risks of several adverse clinical outcomes. Therefore, these patients would warrant a more aggressive approach and early intervention in clinical management to improve prognosis.

\section{Highlights}

Our manuscript is based on the background that the infectious endocarditis (IE) remains a high mortality and is lacking in prognostic predictors for doctors to carry out timely management. We conducted a meta-analysis to prove the association between elevated cardiac troponin level with prognosis in patients with IE which current studies are deficient and no meta-analysis has published in this realm. We attempt to find a more time-efficient, sensitive predictor and provide more effective guidelines for IE management. Our meta-analysis found that patients with higher troponin level in IE were more likely to undergo adverse clinical outcomes, individualized treatment strategy should be set as priority in management of these patients.

\section{Introduction}

Infective endocarditis (IE) is a global disease[1] caused by various pathogenic microorganisms, including streptococcus viridans, staphylococcus aureus, rickettsia, viruses, and some other fungus[2]. This uncommon infection usually affects the native or prosthetic heart valve, endocardial surface, and indwelling cardiac devices[3]. Although the one-year mortality rate of IE has not altered for decades[4], the 
associated morbidity (15/1000000 in the USA) and mortality (10\%-30\% worldwide) remain high[5], and considerably worse than several cancer outcomes.

Appropriate, timely management confers to a better outcome in patients with IE[6]. Although studies have examined several clinical parameters for the prognosis of IE[7], to date, there is a lack of global or multicenter prognostic study, congestive heart failure(CHF) is still the paramount and independent predictive factor for prognosis no matter which type of pathogenic microorganism the patients were infected with[8]. Valve replacement or other expedited cardiac surgeries were usually performed on critical patients in an attempt to achieve a better outcome[9]. Recent studies have demonstrated that older age correlates with a poorer prognosis in IE patients, in addition to other risk factors including cerebral embolization, prosthetic valve endocarditis, and multidrug resistance[10,11]. However, these studies have been limited by their sample size and under-powered to validate confidently the significance of these prognostic indicators.

Troponin complex consists of troponin $\mathrm{C}$ and cardiac-specific troponin I and $\mathrm{T}$, which are specifically located on the thin filament of the myocardium. Troponin regulates the intracellular $\mathrm{Ca} 2+$ concentration that plays a key role in myocardial systolic motion[12]. The measurement of cardiac-specific troponin level allows the identification of myocardial damage, with elevated serum concentration indicating high sensitivity and specificity for the acute coronary syndrome (ACS) compared with other conventional myocardial enzymes. Furthermore, the cardiac troponin level is also a valuable marker for predicting future adverse events in patients with ACS[13]. Interestingly, some studies have also associated the prognostication of cardiac troponin to other non-ACS cardiovascular diseases including IE[14].

A previous study by Watkin et al.[15] in 2004 has demonstrated that patients with high levels of troponin were more likely to undergo valve replacement. In addition, animal studies have revealed elevated levels of troponin in cattles[16] or rhesus[17] that died of IE. However, the available clinical studies to date pertaining to elevated troponin levels and IE prognosis are deficient, especially in sample size and comprehensiveness of analyses in survival prognosis and other clinical outcomes. Therefore, we aimed to systematically review the literature and to conduct a meta-analysis to determine specific clinical outcomes in relation to troponin levels. The findings of the study may provide guidance in the prognostication and management of patients with IE.

\section{Methods}

\subsection{Search strategy}

This study was performed in accordance with the Preferred reporting items for systematic review and meta-analysis(PRISMA)[18]. Literature examining the relationship between cardiac troponin levels and prognostic outcomes in patients with IE were comprehensively searched from Pubmed, Embase, and Cochrane databases on the 14th of July 2020. 'Endocarditis'. 'cardiac troponin', and 'prognosis' were set 
as the subject terms in the medical subject headings (Mesh). Other keywords such as 'infective endocarditis' and 'outcomes' were combined with the subject headings to compose the search strategy.

Following the search, the titles, abstracts, and full-text articles were reviewed and assessed independently by two authors against the inclusion and exclusion criteria. Any disagreements between the reviewers that arose in the process of study selection were resolved with a third reviewer. The risk of bias was also assessed by two authors independently using the Cochrane risk Assessment tool[19, 20], where six domains were judged as 'high risk', 'low risk', or 'unclear' in evaluating the selection, exposure, and comparability in each qualified article.

\subsection{Inclusion and exclusion criteria}

Studies fulfilling the following criteria were included:(1) original articles written in English only and published from the year 2000 to 2020, (2) baseline characteristics of patients were complete, (3) patients were diagnosed with IE according to the Duke criteria[21], (4) elevated cardiac troponin level was set as the risk factor compared with a normal cardiac troponin level as a control, (5) losing of follow up of less than $10 \%$ in RCT. Studies were excluded if: (1) case report, systematic review, or other conference reports, (2) the subjects of research was not human, (3) the original data or the exact number of patients in each group was not accessible, (4) research outcomes were not pertinent to the clinical prognosis of IE.

\subsection{Outcomes}

Only clinical outcomes were incorporated into our meta-analysis, with in-hospital mortality being the most important endpoint. Other endpoints included adverse events of the central nervous system, cardiac abscesses, and the occurrence of valve surgery. Primary and secondary outcomes were specified if stated clearly in the original study. All the outcomes included in the analyses were events that occurred during hospitalization, and any incidences that happened following discharge from the hospital were not recorded or analyzed.

\subsection{Statistical analysis}

Patient baseline characteristics were analyzed with SPSS version 25[22], whereas Revman (review manager, version 5.3) was used to perform the meta-analysis. The association between elevated cardiac troponin level and clinical outcomes was determined with odds ratio (OR) and $95 \%$ confidence interval (Cl). Intervention review, full review stage, and dichotomous variable were assessed on the forest plot.

$\mathrm{Chi}^{2}$ test and $\mathrm{I}^{2}$ were used to assess heterogeneity. $\mathrm{P}<0.05$ in the $\mathrm{Chi}^{2}$ test and $\mathrm{I}^{2}>50$ indicated heterogeneity, where the random-effects model was applied during meta-analysis. whereas the $\mathrm{Chi}^{2}$ test with $P \geq 0.05$ and $I^{2} \leq 50$ indicated homogeneity and thus, the fixed-effects model was utilized[23]. However, when $\mathrm{I}^{2}>50$ but $\mathrm{P} \geq 0.05$ in the $\mathrm{Chi}^{2}$ test, the random-effects model was used. $\mathrm{Z}$ test was utilized for examining the overall effect, with a P-value of $<0.05$ indicating statistical significance in the difference. Publication bias was illustrated in the funnel plot. 
The association between varying locations of focus or lesion in IE and clinical outcome was also explored. The cut-off level of cardiac troponin was also recorded for the detection of potential biases.

The appraisal of study quality and anticipated absolute effects[24] of included outcomes were assessed by using the GRADE[18] (Grading of Recommendations Assessment, Development and Evaluation epro3.3.1)

\section{Results}

\subsection{Study inclusion}

The initial literature search on databases identified 528 articles. After excluding the duplicates, 471 were selected for the further selection process. Of these, 458 were excluded after screening through the titles and abstracts, where the main reasons for exclusion were case or conference report, irrelevant to the objectives of our study, review articles, and animal experiments. A total of 13 studies were selected for full-text screening, following which $4[15,25-27]$ were excluded because of discrepancy in cardiac markers being used (not cardiac troponin) or target population being examined were of other cardiac diseases, 2[28, 29] were eliminated because the original data were not accessible. Ultimately, a total of 7[30-36] studies were included for our meta-analysis (the PRISMA flow diagram was shown in Figure S1(Supplementary file 1)

\subsection{Study and patient baseline characteristics}

The 7 single-center studies included in our meta-analysis were published between 2002 to 2018 and formed a patient pool of 687 in total. Only $2[31,35]$ studies were prospective while others were either retrospective cohort or case-control studies. Among the studies, Ipek et al.[36] and Postigo et al.[33] used cardiac troponin T with cut-off levels of $0.05 \mathrm{ng} / \mathrm{ml}$ and $0.04 \mathrm{ng} / \mathrm{ml}$ respectively as elevated cardiac biomarkers. On the other hand, studies by Tsenovoy et al.[30], Lorson et al.[34], and Thoker et al.[31] used cardiac troponin I as biomarkers with varying cut-off values. Cardiac troponin subtype was however not specified in the studies by Kahveci et al.[35] and Purcell et al.[32] Of 687 patients with IE, most infective lesions were located in the left heart than the right heart with a person-time of 505 versus 213, multiple infections were included in both cohort. There was only 1[31] study conducted in Asia while others were conducted in the USA or Europe. The studies and patient baseline characteristics were shown in Table 1

\subsection{Clinical Outcomes and meta-analysis}

The anticipated absolute effects of each study for different outcomes were outlined in Table 2. Of all the clinical outcomes $(n=10)$ analyzed in our study, in-hospital mortality[37] was rated as 'critical in importance', embolization was rated as 'not important'[38], while other outcomes were assessed as 'important'. As the most important endpoint, in-hospital mortality achieved a risk difference of 279/1000 (95\% Cl 2-526) with elevated cardiac troponin. 
In-hospital mortality was reported in 5[30-34] studies with no obvious heterogeneity detected $\left(\mathrm{Chi}^{2}=\right.$ $2.65, P=0.62 ; I^{2}=0 \%$ ) and thus, fixed-effects model was used. Patients with elevated cardiac troponin had a significantly higher in-hospital mortality than those with a normal level [OR $=5.87,95 \% \mathrm{Cl}$ $(3.37,10.21)]$. Furthermore, $2[33,36]$ studies reported one-year mortality that comprised of a total of 298 patients, which demonstrated an elevated cardiac troponin as a significant risk factor among those diagnosed with IE $[O R=3.28,95 \% \mathrm{Cl}(1.01,10.62)]$. There were $2[30,35]$ studies assessing early surgery and in-hospital mortality together in 105 patients, showing that patients with elevated cardiac troponin were associated with higher mortality or incidence of early surgery during hospitalization [OR $=8.52,95 \%$ $\mathrm{Cl}(3.31,21.93)]$. Of those excluded studies, $2[28,29]$ that did not have accessible original data also reported a higher mortality rate among patients with a higher cardiac troponin level than those with a normal level, with an OR of $7.0[95 \% \mathrm{Cl}(1.7,28.6)]$ and $3.4[95 \% \mathrm{Cl}(1.8,6.4)]$ respectively.

Incidence of surgery (valve replacement) was assessed by $3[33,34,36]$ studies comprised of a total of 510 patients. A higher proportion of patients $(144 / 286,50.35 \%)$ in the group of elevated cardiac troponin levels had undergone surgery, compared with $18.30 \%(41 / 224)$ of patients in the group of normal cardiac troponin level $[\mathrm{OR}=2.18,95 \% \mathrm{Cl}(1.36,3.51)]$.

Symptoms of the central nervous system were assessed in 3[31-33] studies. A significantly higher proportion of patients with an elevated cardiac troponin level $(21.43,54 / 252)$ had symptoms of the central nervous system than those with a normal level $(4.00 \%, 3 / 75)[\mathrm{OR}=3.28,95 \% \mathrm{Cl}(1.01,10.62)]$.

Besides, cardiac abscesses were examined as an outcome in $3[32,33,36]$ studies, comprised of a total of 262 patients with an elevated cardiac troponin level and 86 with a normal level. The incidence of cardiac abscesses of the two groups was $27.10 \%(71 / 262)$ and $5.81 \%(5 / 86)$ respectively, demonstrating a markedly increase in risk among those with a higher cardiac troponin level [OR $=3.28,95 \% \mathrm{Cl}$ $(1.01,10.62)]$.

Furthermore, the incidence of cerebrovascular events was examined as an endpoint in 1[36] study only, with no association found with the levels of cardiac troponin [OR $=3.10,95 \% \mathrm{Cl}(0.64,14.91)]$. Similarly, no significant association was found between renal failure and embolization (assessed by 2 different studies) with the levels of cardiac troponin. On the other hand, patients with elevated cardiac troponin had a higher risk of heart failure (reported in 2 studies). The detailed analyses of all the clinical outcomes in association with cardiac troponin levels were shown in Fig. 1

Except for cerebrovascular events, all the other outcomes in the included studies were homogenous and thus, the fixed-effects model was applied in the meta-analyses for these outcomes. Apart from the outcome of one-year mortality, all the other clinical outcomes were recorded before hospital discharge.

\subsection{Bias assessment}

Included studies were subjected to bias assessment based on the Cochrane risk Assessment tool. All the studies were regarded as having a low risk for detection bias, attrition bias, and reporting bias. However, given that those included studies were not an RCT, the risk of selection bias and performance bias was 
unclear. The risks of other potential biases were outlined in Figure S2. The GRADE evidence profile demonstrated the quality and risk of bias individually for various outcomes. In 6 studies, the certainty of the evidence was rated as 'very low', while one study was rated as 'low certainty' (Table 2).

For in-hospital mortality, funnel plots revealed uniformity and symmetrical distribution, indicating no significant publication bias among these studies (Figure S3). However, publication bias of other outcomes could not be assessed due to lacking in numbers of included studies.

\section{Discussion}

At present, few studies have explored the association between elevated cardiac troponin and prognosis in patients with IE. Our meta-analysis provided a quantitative summary of the prognostic value of cardiac troponin in various clinical outcomes. Among the 7 included studies, outcomes consisted of in-hospital mortality (5 studies), one-year mortality ( 2 studies), early surgery or in-hospital mortality(2 studies),surgery or valve replacement (3 studies), CNS events (3 studies), cardiac abscess (3 studies), renal failure (2 studies), heart failure ( 2 studies), embolization ( 2 studies), and cerebrovascular event (1 study) were examined in relation to the level of cardiac troponin. No significant correlation was found between elevated cardiac troponin and embolization, renal failure, or cerebrovascular events However, Elevated cardiac troponin in patients with IE was observed to correlate significantly to higher risks of inhospital or one-year mortality, surgery or valve replacement, CNS events, cardiac abscess, and heart failure, indicating that patients with IE and elevated cardiac troponin level demand a closer observation and more prudent intervention.

For patients with IE, the risk of in-hospital mortality represents the most important and commonly reported outcome[39], with all 5 studies[30-34] suggested the need to pay more attention in the management of IE patients with elevated cardiac troponin, given that these patients may have a poorer survival prognosis or higher risk of other complications than those with normal cardiac troponin. The review article by Burton et al.[40] in 2013 included 5 studies, which explored the prognostic value of cardiac troponin levels in patients with IE. In the study, the proportions of patients with increased serum levels of cardiac troponin were estimated to range from $57-73 \%$. The authors summarized the included studies individually and claimed that an increased cardiac troponin level was associated with mortality without a systematic review and meta-analysis. Coupled with their postulation that a non-elevated cardiac troponin level might be more valuable in predicting prognosis has prompted our present study.

The studies by Mills et al.[41] and Baddour et al.[6] have shown that heart failure, valvular rupture or fistula, and CNS events are associated with poor prognosis in IE. These outcomes are all accompanied by a myocardial injury that releases cardiac troponin, corresponding with our findings that an elevated cardiac troponin confers to a higher risk of surgery or valve replacement, cardiac abscess, heart failure, and CNS events. On the other hand, renal failure and embolization are consequences of bacteremia and vegetation[42], which have no direct association with myocardial damage and therefore, support our 
findings that elevated troponin has no significant correlation with risk of embolization, renal failure, or cerebrovascular events.

The N-terminal pro-B-type natriuretic peptide (NT-pro-BNP) may act as a prognostic factor for IE[35] and recent studies have demonstrated that an elevated natriuretic peptide level to a higher risk of in-hospital death in IE patients[43]. However, cardiac troponin was not included as part of the analyses in these studies. Similarly, our included studies also have not incorporated other serum biomarkers that may play an integral part in interfering with the level of cardiac troponin and predicting the poor outcomes.

Early surgery has been advocated for patients with solid indications for such intervention[44], but studies have also shown that surgery may increase the risk of 6-month mortality, given that it is always performed for patients with a severe form of IE which is associated with severe complications despite it achieving higher survival rate than palliative treatment. From our study, it is unclear if the increase in mortality risk was correlated with an elevated cardiac troponin independently or was confounded by surgical intervention that resulted in the release of cardiac troponin.

Our study is the first meta-analysis to examine the association and predictive value of cardiac troponin for several outcomes in patients with IE. However, further studies are warranted to investigate the longterm outcomes associated with an elevated troponin level. The pathophysiological mechanism and doseeffect relationship should also be examined which will provide guidance for patient management.

\section{Limitations}

All the included studies were of single-center cohorts. The number of studies included and sample sizes in each study were small. Most studies (6/7) were conducted in America and Europe, of which the findings may not be applicable to Asian patients. Also, variable cut-off values of cardiac troponin were used in the studies to indicate an elevated serum troponin concentration, which may result in result deviation. Moreover, confounding factors such as incomplete data on baseline characteristics or inaccessible to some of the original data may have an adverse impact on our analyses.

\section{Conclusion}

Our study has shown that IE patients with an elevated cardiac troponin level may have a poorer prognosis, particularly associated with higher risks of in-hospital mortality, early surgery or valve replacement, cardiac abscesses, and heart failure. These findings highlight the importance of an individualized treatment strategy for patients with elevated cardiac troponin in the management of IE.

\section{Abbreviations}

Infective endocarditis IE

congestive heart failure CHF 
acute coronary syndrome ACS

reporting items for systematic review and meta-analysis PRISMA

odds ratio $\mathrm{OR}$

confidence interval $\mathrm{Cl}$

\section{Declarations}

\section{Ethics approval and consent to participate}

Not applicable. This systematic review and meta-analysis did not involve research on any human subjects requiring informed consent.

\section{Consent for publication}

Not applicable.

\section{Availability of data and materials}

All relevant data has been provided in figures, tables and text.

\section{Competing interests}

There are no financial disclosures or conflicts of interest for any of the authors.

\section{Funding}

The Scientific Research Project of National Clinical Research Center for Geriatrics $\llbracket$ West China Hospital, Sichuan University (No. Z2018B19), and 1.3.5 project for disciplines of excellence-Clinical Research Incubation Project, West China Hospital, Sichuan UniversityهNo. 2019HXFH029囚, and Technology innovation research and development project of Chengdu Science and Technology Bureau『No. 2019YF05-00183-SN区, and the Major Science and Technology Project of Sichuan Province, China (No. 2021YFS0121);

\section{Authors' contributions}

Study concept: Zheng Chai and Yunfei Ling. Study design: Zheng Chai and Yunfei Ling. Data acquisition: Zheng Chai and Yongjun Qian. Quality control of data and algorithms: Zheng Chai and Tiange Li. Data analysis: Zheng Chai and Zhongze Cao. Manuscript preparation: Zheng Chai, Tiange Li and Kerun Chen. Manuscript editing: Yongjun Qian, Zheng Chai and Yunfei Ling. Manuscript review: Zheng Chai and Yunfei Ling. All the authors have read and approved the final version of the manuscript.

\section{Acknowledgements}


None.

\section{References}

1. Cahill TJ, Prendergast BD: Infective endocarditis. Lancet 2016, 387(10021):882-893.

2. Tleyjeh IM, Steckelberg JM: Changing epidemiology of infective endocarditis. Curr Infect Dis Rep 2006, 8(4):265-270.

3. Yang E, Frazee BW: Infective Endocarditis. Emerg Med Clin North Am 2018, 36(4):645-663.

4. Knebel F, Frumkin D, Flachskampf FA: [Infective Endocarditis]. Dtsch Med Wochenschr 2019, 144(2):114-127.

5. Wang A, Gaca JG, Chu VH: Management Considerations in Infective Endocarditis: A Review. Jama 2018, 320(1):72-83.

6. Baddour LM, Wilson WR, Bayer AS, Fowler VG, Jr., Tleyjeh IM, Rybak MJ, Barsic B, Lockhart PB, Gewitz MH, Levison ME et al: Infective Endocarditis in Adults: Diagnosis, Antimicrobial Therapy, and Management of Complications: A Scientific Statement for Healthcare Professionals From the American Heart Association. Circulation 2015, 132(15):1435-1486.

7. Management of infective endocarditis. Drug Ther Bull 2002, 40(4):26-30.

8. Sexton DJ, Spelman D: Current best practices and guidelines. Assessment and management of complications in infective endocarditis. Cardiol Clin 2003, 21(2):273-282, vii-viii.

9. Razmi R, Magnusson P: [Management of infective endocarditis]. Lakartidningen 2019, 116.

10. Nishimura RA, Otto CM, Bonow RO, Carabello BA, Erwin JP, 3rd, Guyton RA, O'Gara PT, Ruiz CE, Skubas NJ, Sorajja P et al: 2014 AHAACC guideline for the management of patients with valvular heart disease: a report of the American College of Cardiology/American Heart Association Task Force on Practice Guidelines. J Am Coll Cardio/ 2014, 63(22):e57-185.

11. Drew RH: Emerging options for treatment of invasive, multidrug-resistant Staphylococcus aureus infections. Pharmacotherapy 2007, 27(2):227-249.

12. Katrukha IA: Human cardiac troponin complex. Structure and functions. Biochemistry (Mosc) 2013, 78(13):1447-1465.

13. Jarolim P: High sensitivity cardiac troponin assays in the clinical laboratories. Clin Chem Lab Med 2015, 53(5):635-652.

14. Eggers KM, Lindahl B: Application of Cardiac Troponin in Cardiovascular Diseases Other Than Acute Coronary Syndrome. Clin Chem 2017, 63(1):223-235.

15. Watkin RW, Lang S, Smith JM, Elliott TS, Littler WA: Role of troponin I in active infective endocarditis. Am J Cardiol 2004, 94(9):1198-1199.

16. Mellanby RJ, Henry JP, Cash R, Ricketts SW, Bexiga R, Truyers I, Mellor DJ: Serum cardiac troponin I concentrations in cattle with cardiac and noncardiac disorders. J Vet Intern Med 2009, 23(4):926930 . 
17. Zafalon-Silva B, Soares FAC, Pavarini SP, Goulart MA, Snel GGM, González FHD, Vaz DB, Lopes BC, Oliveira M, Alievi MM: Bacterial endocarditis and increased cardiac troponin I levels in a brown howler monkey (Alouatta guariba clamitans) with an interventricular septal defect. J Med Primatol 2019, 48(2):129-132.

18. Moher D, Shamseer L, Clarke M, Ghersi D, Liberati A, Petticrew M, Shekelle P, Stewart LA: Preferred reporting items for systematic review and meta-analysis protocols (PRISMA-P) 2015 statement. Syst $\operatorname{Rev} 2015,4(1): 1$.

19. Lo CK, Mertz D, Loeb M: Newcastle-Ottawa Scale: comparing reviewers' to authors' assessments. BMC Med Res Methodol 2014, 14:45.

20. Higgins JP, Altman DG, Gøtzsche PC, Jüni P, Moher D, Oxman AD, Savovic J, Schulz KF, Weeks L, Sterne JA: The Cochrane Collaboration's tool for assessing risk of bias in randomised trials. $\mathrm{Bmj}$ 2011, 343:d5928.

21. Naber CK, Bartel T, Eggebrecht H, Erbel R: [Diagnosis of endocarditis today: Duke criteria or clinical judgment?]. Herz 2001, 26(6):379-390.

22. Gouda MA: Common Pitfalls in Reporting the Use of SPSS Software. Med Princ Pract 2015, 24(3):300.

23. DerSimonian R, Laird N: Meta-analysis in clinical trials revisited. Contemporary clinical trials 2015, 45(Pt A):139-145.

24. Venekamp RP, Rovers MM, Hoes AW, Knol MJ: Subgroup analysis in randomized controlled trials appeared to be dependent on whether relative or absolute effect measures were used. Journal of clinical epidemiology 2014, 67(4):410-415.

25. Ryu HM, Bae MH, Lee SH, Lee JH, Kwon YS, Yang DH, Park HS, Cho Y, Chae SC, Jun JE et al: Presence of conduction abnormalities as a predictor of clinical outcomes in patients with infective endocarditis.

26. Navarro-Navarro A, Castillo-Martinez L, Orea-Tejeda JIA, Carrasco-Ortiz O, Sanchez-Santillan R, Sanchez-Vidal G, Pineda-Juarez J, Vazquez-Duran M, Gutierrez-Rodriguez A, Gaspariano-Flores E: High cardiac troponin I concentrations are associated with worse outcome instable heart failure patients.

27. Watkin RW: Myocardial involvement in infective endocarditis: can serum troponin levels predict the outcome? Cardiology 2009, 112(3):200-201.

28. Siciliano RF, Gualandro DM, Sommer Bittencourt M, Paixão M, Marcondes-Braga F, de Matos Soeiro A, Strunz C, Pacanaro AP, Puelacher C, Tarasoutchi F et al: Biomarkers for prediction of mortality in left-sided infective endocarditis. Int J Infect Dis 2020, 96:25-30.

29. Stancoven AB, Shiue AB, Khera A, Pinkston K, Hashim IA, Wang A, de Lemos JA, Peterson GE: Association of troponin $\mathrm{T}$, detected with highly sensitive assay, and outcomes in infective endocarditis. Am J Cardio/ 2011, 108(3):416-420.

30. Tsenovoy P, Aronow WS, Joseph J, Kopacz MS: Patients with infective endocarditis and increased cardiac troponin I levels have a higher incidence of in-hospital mortality and valve replacement than 
those with normal cardiac troponin I levels. Cardiology 2009, 112(3):202-204.

31. Thoker ZA, Khan KA, Rashid I, Zafar: Correlation of cardiac troponin I levels with infective endocarditis \& its adverse clinical outcomes. Int J Cardio/ 2016, 222:661-664.

32. Purcell JB, Patel M, Khera A, de Lemos JA, Forbess LW, Baker S, Cabell CH, Peterson GE: Relation of troponin elevation to outcome in patients with infective endocarditis. Am J Cardio/2008, 101(10):1479-1481.

33. Postigo A, Bermejo J, Muñoz P, Valerio M, Marín M, Pinilla B, Cuerpo G, Marí A, Delgado-Montero A, Bouza $\mathrm{E}$ et al: Troponin elevation is very common in patients with infective endocarditis and is associated with a poor outcome. Int J Cardiol 2020, 307:82-86.

34. Lorson W, Veve MP, Heidel E, Shorman MA: Elevated troponin level as a predictor of inpatient mortality in patients with infective endocarditis in the Southeast United States. BMC Infect Dis 2020, 20(1):24.

35. Kahveci G, Bayrak F, Mutlu B, Bitigen A, Karaahmet T, Sonmez K, Izgi A, Degertekin M, Basaran Y: Prognostic value of $\mathrm{N}$-terminal pro-B-type natriuretic peptide in patients with active infective endocarditis. Am J Cardio/2007, 99(10):1429-1433.

36. Gucuk Ipek E, Guray Y, Acar B, Kafes H, Dinc Asarcikli L, Cabuk G, Demirkan B, Kuyumcu MS, Guray U: The usefulness of serum troponin levels to predict 1-year survival rates in infective endocarditis. Int $J$ Infect Dis 2015, 34:71-75.

37. Selton-Suty C, Goehringer F, Venner C, Thivilier C, Huttin O, Hoen B: [Complications and prognosis of infective endocarditis]. Presse Med 2019, 48(5):532-538.

38. Bitay M, Varga S, Babik B, Havasi K, Szücsborus T: Infective endocarditis complicated with coronary artery septic embolization: is it worth to be mentioned? Case presentation and review of the literature. Rev Cardiovasc Med 2019, 20(1):35-39.

39. Abegaz TM, Bhagavathula AS, Gebreyohannes EA, Mekonnen AB, Abebe TB: Short- and long-term outcomes in infective endocarditis patients: a systematic review and meta-analysis. BMC CardiovasC Disord 2017, 17(1):291.

40. Burton MJ, Cockrell J, Holguin G, Geraci S: Prognostic value of cardiac troponin levels in infective endocarditis. Ther Adv Cardiovasc Dis 2013, 7(1):45-46.

41. Mills $\mathrm{J}$, Utley $\mathrm{J}$, Abbott $\mathrm{J}$ : Heart failure in infective endocarditis: predisposing factors, course, and treatment. Chest 1974, 66(2):151-157.

42. Liu J, Frishman WH: Nonbacterial Thrombotic Endocarditis: Pathogenesis, Diagnosis, and Management. Cardiol Rev 2016, 24(5):244-247.

43. Mistiaen WP: Hospital mortality and its predictors in patients with infective endocarditis. $J$ Cardiovasc Surg (Torino) 2018, 59(2):302-303.

44. The 2015 ESC Guidelines for the management of infective endocarditis. Eur Heart J 2015, 36(44):3036-3037. 


\section{Tables}

Due to technical limitations, table 1 and 2 is only available as a download in the Supplemental Files section.

\section{Figures}
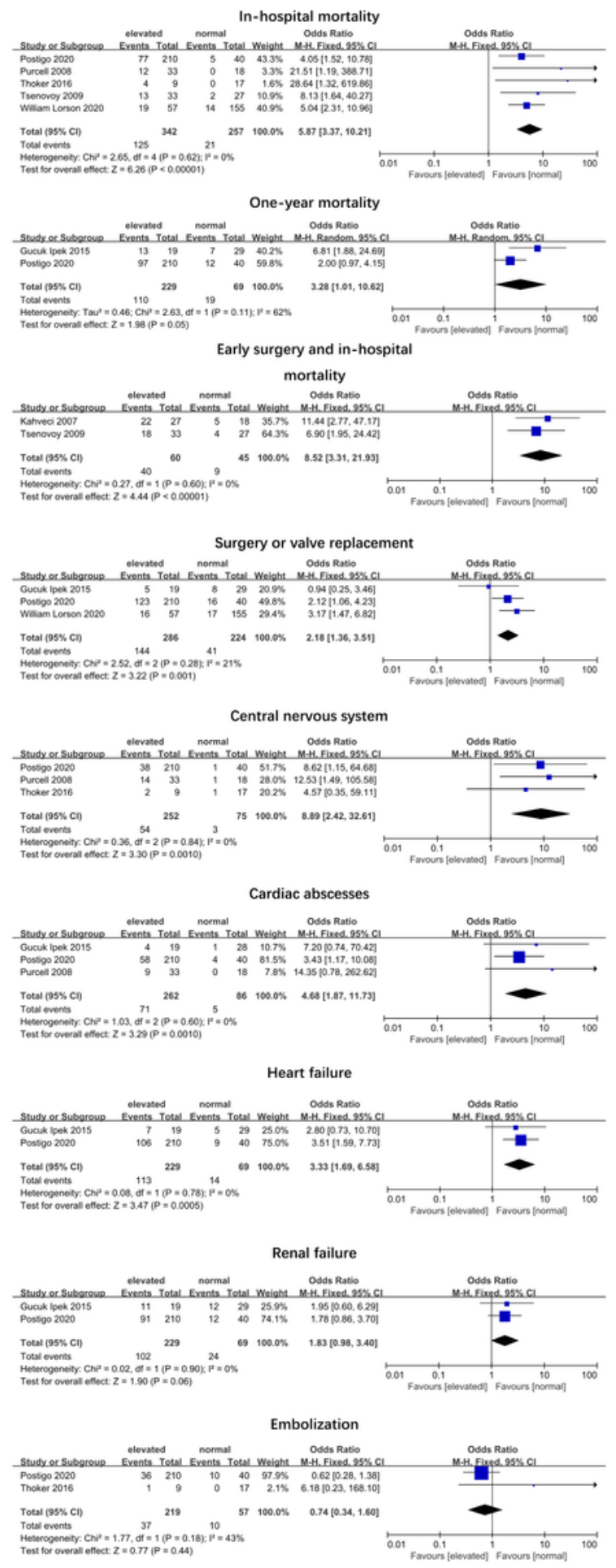
Figure 1

Forest plot of different clinical outcomes

\section{Supplementary Files}

This is a list of supplementary files associated with this preprint. Click to download.

- FigureS1.png

- Figures2.png

- FigureS3.png

- Table1.png

- Table2.png

- PRISMA2009checklist.doc 